\title{
Coordinated control for medium voltage DC distribution centers with flexibly interlinked multiple microgrids
}

\author{
Lei QIAO ${ }^{1}$, Xialin LI $^{1}$, Di HUANG ${ }^{1}$, Li GUO ${ }^{1}$, Yutao $\mathrm{XU}^{2}$, \\ Zhukui TAN $^{2}$, Chengshan WANG ${ }^{1}$
}

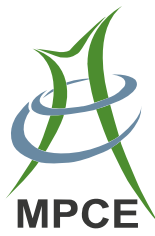

\begin{abstract}
From flexible interconnection among feeders to hybrid alternating current (AC) and direct current (DC) distribution structures of future smart distribution systems, medium-voltage DC distribution centers with flexibly interlinked multiple microgrids (MGs) will have wide applications on the demand side. A generic coordinated control framework based on a distributed cooperation scheme is proposed for such DC centers, as opposed to centralized control structures. A novel unified control only using local measurements is proposed for these interlinking converters. During normal power disturbances, automatic coordinated power control and mutual support among sub-
\end{abstract}

CrossCheck date: 16 August 2018

Received: 20 December 2017 / Accepted: 16 August 2018/Published online: 25 October 2018

(C) The Author(s) 2018

$\triangle$ Xialin LI

xialinlee@tju.edu.cn

Lei QIAO

qiaolei@tcu.edu.cn

Di HUANG

huangdi06@tju.edu.cn

Li GUO

liguo@tju.edu.cn

Yutao XU

95616048@qq.com

Zhukui TAN

tanzk@163.com

Chengshan WANG

cswang@tju.edu.cn

1 The Key Laboratory of Smart Grid of Ministry of Education, Tianjin University, Tianjin 300072, China

2 Electric Power Research Institute, Guizhou Power Grid Co. Ltd., Guiyang 550002, China systems can be realized, thereby improving DC voltage and AC frequency stability to enable multiple MGs to be treated as a real unified cluster. Moreover, with this method, interlinking converters can realize seamless transition in power dispatching mode, common DC bus voltage control mode, and MG support mode without communication and control system switching. A simplified dynamic model has been developed to verify the proposed control strategy. This work is expected to provide a new solution for flexible interconnection and operational control of large-scale MG clusters.

Keywords Medium-voltage DC distribution centers, Multiple microgrids, Flexible interconnection, Coordinated control, Seamless transition

\section{Introduction}

With large-scale distributed generations (DGs), energy storage systems (ESSs), electric vehicles (EVs), and multiple microgrids (MGs) integrated with distribution systems, and with the increasing implementation of novel technologies (e.g., active distribution, self-healing, and demand-side response) a tie switch-based direct connection structure among different alternating current (AC) feeders or stations has become a major bottleneck limiting improvements to flexibility, reliability, and economy of distribution systems. Moreover, future distribution systems aim to achieve more flexible, reliable, and efficient network architectures and power-flow scheduling and control $[1,2]$. If traditional tie switches could be replaced by voltagesource converter-based back-to-back intelligent soft open point (SOP) [3-5], flexible interconnection and accurate power-flow control among feeders and stations could be 
realized. This has recently become a widespread concern in both academia and industry.

With the development of power semiconductors and power electronic technologies, and with increasing applications of direct current (DC) loads, DC distribution systems, with their strong power supply capability, low cost, high efficiency, and reliability [6-8], have gradually become a hot research topic. In China, many research institutes, universities, and enterprises have participated in projects on hybrid AC-DC MGs with high density DGs, hybrid AC-DC distribution systems, and flexible mediumvoltage DC power distributions [9, 10]. A technical construction scheme demonstration of a $\pm 10 \mathrm{kV}$ multi-terminal DC distribution project in Shenzhen was proposed in [11]. The authors in [12] presented an architecture for an AC-DC seamless hybrid flexible power distribution system based on novel fully controllable flexible distribution equipment. In [13], DC distribution voltage level sequences, typical network architectures, and application scenarios were discussed in detail, revealing that DC distribution technologies could provide innovative ideas for future intelligent distribution networks.

In medium voltage distribution levels, it can be expected that future smart distribution systems will experience flexible interlinking stages based on SOP technology. Then, gradually, more flexible, reliable, and efficient ACDC distribution networks can be produced. As an extant and important transition form of intelligent distribution system, medium-voltage DC distribution centers (MVDCs) effectively and deeply integrate multi-terminal SOPs and AC-DC MGs on the demand side [14-16], allowing flexible interconnection of multiple $\mathrm{AC}$ feeders and interaction with the AC-DC MGs. Such MVDCs will have wide application in urban heavy-load centers with limited increased capacity of AC distribution networks and multiterminal flexible DC distribution networks [13].

This paper focuses on flexible operation and control of MVDCs with multiple MGs. A hierarchical control-based traditional framework is always adopted [16-18]. With primary controls, DC voltage control related to medium voltage DC bus and DC MGs, AC voltage/frequency control related to $\mathrm{AC}$ MGs are realized via individual local controllers, which are related to short-term control timescales. Coordinated control and optimal operation over the long-term are implemented in secondary control or higher level. $P-f$ and $P-U_{\mathrm{dc}}$ droop controllers [17-20] are preferable local controllers for slack terminals in $\mathrm{AC}$ and $\mathrm{DC}$ MGs and for DC-AC converters connected to an AC distribution system. For MG interlinking converters, three basic control modes (i.e., power dispatching mode, common DC bus voltage control mode, and MG support mode) can be adopted under different operation situations. Specific local controller designs for these interlinking converters play a very important role in control stability and flexibility for DC centers in the short-term. However, for different control modes, different control systems are always specifically designed. Consequently, with unscheduled transients, such as lost or faulty slack terminals in MGs, seamless transition among different operation modes without communication becomes a tough issue. Another important consideration when designing interlinking converter control strategies is realizing fast coordinated power control and mutual support among subsystems under normal disturbances by using only locally measured signals. Motivated by the above mentioned issues at the primary control level, a novel unified control using only local measurements is proposed for these interlinking MG converters.

The main contributions of this paper are as follows:

1) A generic coordinated control framework, based on a distributed cooperation scheme, is presented to improve DC-center control stability and flexibility over the centralized operation structures. Each subsystem has an individual central controller to coordinate the local controllers, achieving autonomous operational target. Moreover, via flexible interconnection of converters and distributed communications, distributed optimal operation amongst all sub-regions can be realized.

2) A novel unified control that only uses locally measured signals is proposed for the MG's interlinking converters. Via this method, coordinated power control and mutual support among sub-systems can be realized automatically during normal power disturbances, improving DC voltage and AC frequency stability, allowing multiple MGs to be treated as a unified cluster. Furthermore, seamless transition among power dispatching mode, common DC bus voltage control mode, and $\mathrm{MG}$ support mode of interlinking converters can be realized without communication and control system switching.

3) A simplified dynamic model, including dynamic features of physical layers and local controllers of the considered MVDC is developed to validate the proposed coordinated control scheme.

This work is expected to provide a new solution for flexible interconnection and operation control of largescale MG clusters. The rest of the paper is organized as follows. Section 2 presents configuration and operation mode analysis of the considered MVDC. The proposed coordinated controls and their detailed analysis are presented in Section 3. Section 4 provides simulation validation of the proposed method. Finally, Section 5 concludes this paper. 


\section{Configuration and operation mode analysis of considered MVDC}

\subsection{System configuration}

Figure 1 presents the considered MVDC, including an AC MG, a DC MG, and a medium-voltage DC bus, where PCS stands for power converter system. More MGs can be connected to the DC center, provided the MVDC capacity is large enough. As seen in Fig. 1, the MVDC is connected to the AC distribution system through $N_{\mathrm{MV}}$ DC-AC bidirectional converters. Using a multi-terminal structure, not only can the MVDC power supply reliability be enhanced, but flexible interlinking among multiple feeders of the AC distribution system can also be realized. Renewable energy sources based DGs, ESSs and loads can be connected to AC and DC MGs. The units within MGs can be recognized as slack terminals and power terminals. Here, the slack terminals mean the DC voltage control units and AC voltage/frequency control units, like controllable DGs or ESSs. Renewable energy sources based DGs with maximum power tracking control, DGs or ESSs under power dispatch mode, and loads can all be viewed as power terminals. Without loss of generality, $N_{\mathrm{ac}}$ and $N_{\mathrm{dc}}$ slack terminals are considered in AC and DC MGs. To be flexibly interlinked to the MVDC, bi-directional DC-AC and DCDC interlinking converters have been used for $\mathrm{AC}$ and $\mathrm{DC}$ MGs, respectively.

\subsection{Operation mode analysis}

In terms of the actual conditions of the AC distribution system and MG slack terminals, the considered MVDC has multiple operation modes. To conveniently analyze the operation modes and related controls, the following Mode 1 (normal operation) is considered as the basic operation mode.

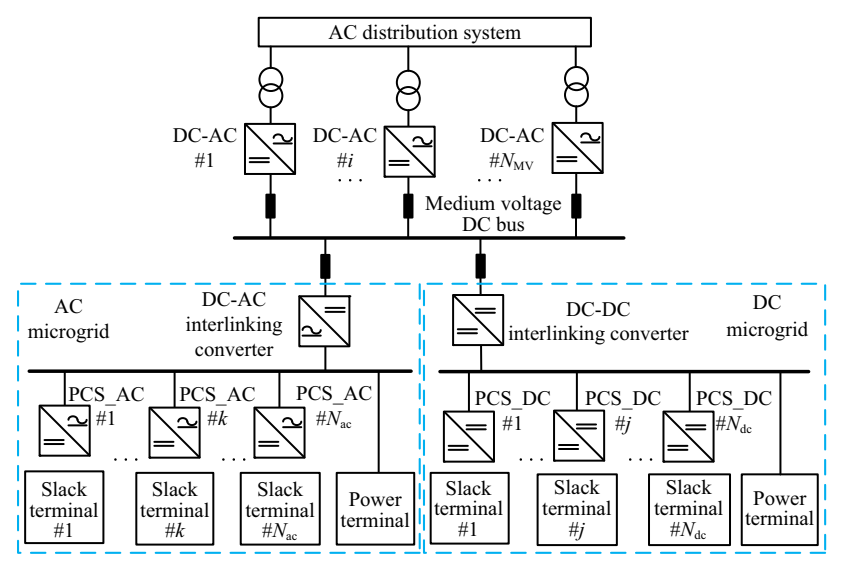

Fig. 1 Configuration of considered MVDC
1) Mode 1 (normal operation)

In this mode, the medium DC bus voltage is controlled by DC-AC \#1 to $\# N_{\mathrm{MV}}$. The DC voltage in the DC MG and AC voltage/frequency in the AC MG are controlled by their slack terminals and related converters respectively. It should be noted that no specific control strategy (e.g., master-slave or peerpeer control) has been determined here. However, only subjects and functions of the mentioned controls are emphasized. These DC-AC and DC-DC interlinking MG converters work in a power control mode to regulate power flows between MGs and mediumvoltage DC buses with power dispatching references received from upper control levels.

This work addresses the important issue of achieving automatically coordinated power control and mutual support amongst sub-systems with only locally measured signals to improve DC voltage and AC frequency stability and to enable multiple MGs to behave as a unified cluster under power disturbances.

Based on the normal operation mode, other MVDC operation modes can be grouped by MGs supported by main grid (Mode 2) and islanded operation or mutual support of AC and DC MGs (Mode 3) with detailed analysis as follows.

2) Mode 2 (microgrid supported by main grid)

Suppose that if slack terminals in AC MG or DC MG are limited to output or even become unavailable in case of abnormal situations, such as faults or insufficient reserve capacity, the AC MG or DC MG will lose its power balancing capability, rendering AC voltage /frequency or DC voltage uncontrollable. In these scenarios, these interlinking MG converters are expected to take charge of power balancing. Then, the AC distribution system can provide power support and stable DC voltage (AC frequency) control to the faulty MG through the medium-voltage DC bus, which is called as microgrid supported by main grid mode in this work.

3) Mode 3 (islanded operation or mutual support of AC and DC microgrids)

When the medium DC voltage cannot be controlled stably by DC-AC \#1 to \# $N_{\mathrm{MV}}$ due to faults in the AC distribution system, the AC and DC MGs can choose to work in an islanded mode in mutual support of AC and DC MGs. Moreover, with an appropriate power control strategy for the interlinking converters, multiple MGs can operate interlinked in a mutual support mode.

In summary, the DC-AC and DC-DC interlinking converters have different control targets in different operation modes. An important issue addressed by this paper is realizing seamless transition among power 
dispatching mode, common DC bus voltage control mode, and MG support mode of interlinking converters without communication and control system switching.

\section{Proposed MVDC coordinated control}

\subsection{Basic framework of proposed coordinated control for MVDC}

To improve the control stability and flexibility of the considered MVDC, a generic coordinated control framework based on autonomous control of sub-system and coordinated control among all sub-regions is proposed, as shown in Fig. 2. With the proposed coordinated control, the MVDC is expected to achieve the following goals.

1) Each sub-system, including the medium voltage $\mathrm{DC}$ bus and the AC and DC MGs, can be controlled via their individual hierarchal control systems, consisting of local control for the physical layer units and uppercentral control, which enable the sub-system to operate in autonomous mode. Moreover, coordinated optimal control among sub-regions can be realized via distributed cooperation of these central controllers, consisting of global optimization and regional optimization, which will be discussed briefly in the next section.

2) All local controllers are designed to be unified. Thus, control stability and flexibility of each sub-system can be improved. With unified local controllers, automatic coordinated power control and mutual support among sub-systems under normal disturbances can be realized using local measurements. Moreover, these MG interlinking converters can achieve seamless transition among power dispatching mode, common DC busvoltage control mode, and MG support mode without communication and control system switching.

3) Through distributed optimal power dispatching, the MVDC can achieve a planned optimal operation target. Compared to centralized optimization, the distributed cooperation framework shown in Fig. 2 will enhance flexibility and scalability.

\subsection{Local control design for slack terminals}

In Mode 1, as described in Section 2.2, the main function of local control of slack terminals in each sub-region is to control DC voltage in the DC MG and the mediumvoltage $\mathrm{DC}$ bus and the $\mathrm{AC}$ frequency/voltage in the $\mathrm{AC}$ MG. This includes maintaining a power balance in this region. The droop method [17-20] has been used for local control of slack terminals in medium-voltage DC bus, the DC MG, and the AC MG, as shown in Fig. 3a-c, respectively.

With droop control, the DC voltages and AC frequency in the MVDC have the following droop features in (1), where all variables are in p.u. values.

$$
\left\{\begin{array}{l}
u_{\mathrm{MV}}=u_{i, \mathrm{MV}}^{*}-P_{\mathrm{s} i, \mathrm{MV}} / k_{i, \mathrm{MV}} \\
u_{\mathrm{dc}}=u_{j, \mathrm{c}}^{*}-P_{\mathrm{s} j, \mathrm{dc}} / k_{j, \mathrm{dc}} \\
\omega_{\mathrm{ac}}=\omega_{k, \mathrm{ac}}^{*}-P_{\mathrm{s} k, \mathrm{ac}} / k_{k, \mathrm{ac}}
\end{array}\right.
$$

where $u_{\mathrm{MV}}$ is the actual medium DC voltage; $u_{i, \mathrm{MV}}^{*}, P_{\mathrm{s} i, \mathrm{MV}}$ and $k_{i, \mathrm{MV}}$ are the DC voltage setting value, power injected

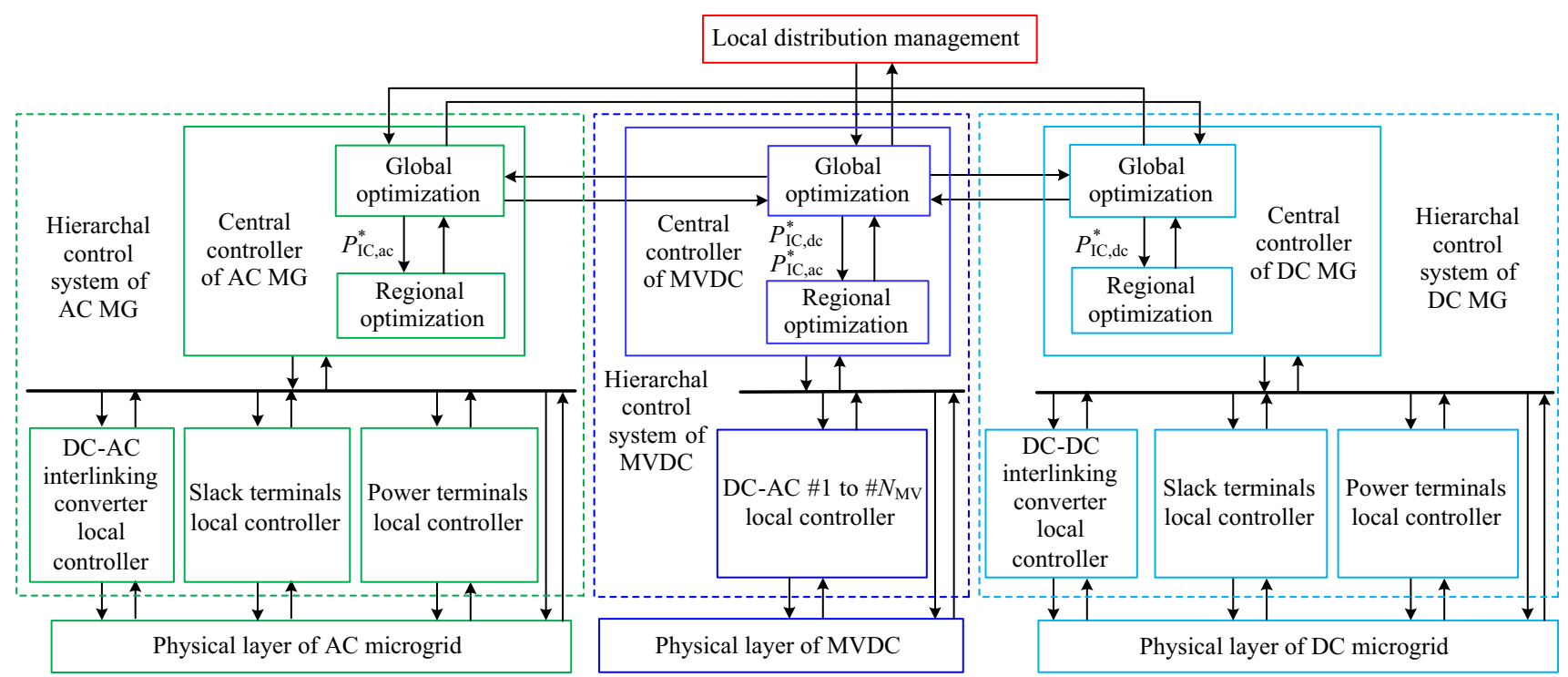

Fig. 2 Generic framework of proposed coordinated control 


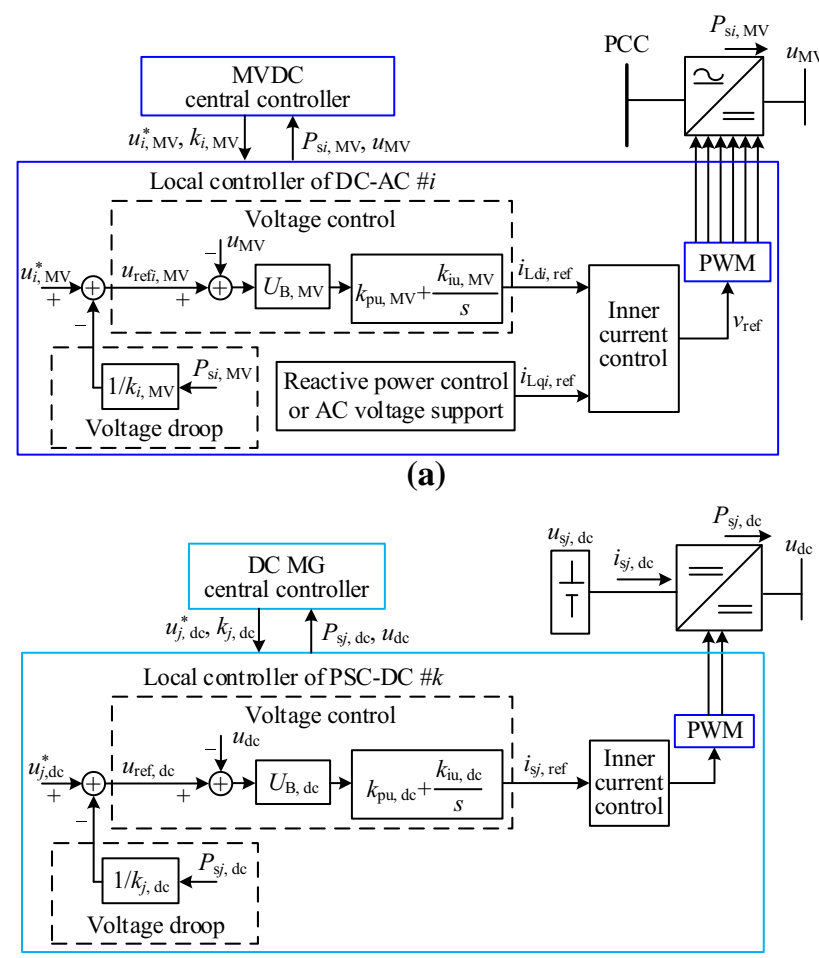

(b)

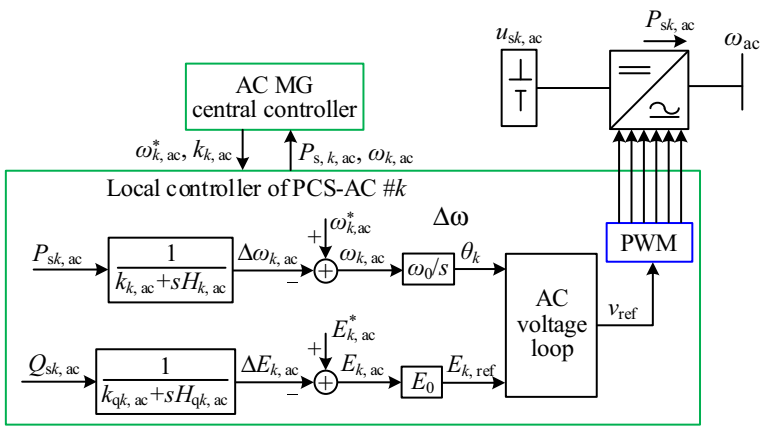

(c)

Fig. 3 Local control schemes for the slack terminals in MVDC

into the medium voltage DC bus, and droop gain of the $i^{\text {th }}$ slack terminal in the medium-voltage DC bus, respectively; $u_{\mathrm{dc}}$ is the actual DC voltage in the DC MG; $u_{j, \mathrm{dc}}^{*}, P_{\mathrm{sj} j \mathrm{dc}}$, and $k_{j, \mathrm{dc}}$ are the DC voltage setting values, power injected into DC bus, and droop gain of the $j^{\text {th }}$ slack terminal in DC MG, respectively; $\omega_{\mathrm{ac}}$ is the actual $\mathrm{AC}$ frequency in the $\mathrm{AC}$ MG; $\omega_{k, \mathrm{ac}}^{*}, P_{\mathrm{s} k, \mathrm{ac}}$, and $k_{k, \mathrm{ac}}$ are the $\mathrm{AC}$ frequency setting value, active power injected into $\mathrm{AC}$ bus, and droop gain of the $k$ th slack terminal in the AC MG, respectively. It should be noted that these droop control parameters (i.e., $u_{i, \mathrm{MV}}^{*}$ and $k_{i, \mathrm{MV}}, u_{j, \mathrm{dc}}^{*}, k_{j, \mathrm{dc}}, \omega_{k, \mathrm{ac}}^{*}$, and $\left.k_{k, \mathrm{ac}}\right)$ can be tuned via optimal control in central controllers to realize optimal power redistribution of slack terminals. For further analysis, an aggregated slack terminal in each sub-system is derived with its equivalent droop feature, deduced as:
$\left\{\begin{array}{l}u_{\mathrm{MV}}=u_{\mathrm{MV}}^{*}-P_{\mathrm{s}, \mathrm{MV}} / k_{\mathrm{MV}} \\ u_{\mathrm{dc}}=u_{\mathrm{dc}}^{*}-P_{\mathrm{s}, \mathrm{dc}} / k_{\mathrm{dc}} \\ \omega_{\mathrm{ac}}=\omega_{\mathrm{ac}}^{*}-P_{\mathrm{s}, \mathrm{ac}} / k_{\mathrm{ac}}\end{array}\right.$

where equivalent DC voltage (AC frequency) settings, aggregated power injections, and droop gains in (2) can be obtained as:

$u_{\mathrm{MV}}^{*}=\sum_{i=1}^{N_{\mathrm{MV}}}\left(u_{i, \mathrm{MV}}^{*} k_{i, \mathrm{MV}}\right) / \sum_{i=1}^{N_{\mathrm{MV}}} k_{i, \mathrm{MV}}$

$P_{\mathrm{s}, \mathrm{MV}}=\sum_{i=1}^{N_{\mathrm{MV}}} P_{\mathrm{si}, \mathrm{MV}}$

$k_{\mathrm{MV}}=\sum_{i=1}^{N_{\mathrm{MV}}} k_{i, \mathrm{MV}}$

$u_{\mathrm{dc}}^{*}=\sum_{j=1}^{N_{\mathrm{dc}}}\left(u_{j, \mathrm{dc}}^{*} k_{j, \mathrm{dc}}\right) / \sum_{j=1}^{N_{\mathrm{dc}}} k_{j, \mathrm{dc}}$

$P_{\mathrm{s}, \mathrm{dc}}=\sum_{j=1}^{N_{\mathrm{dc}}} P_{\mathrm{s} j, \mathrm{dc}}$

$k_{\mathrm{dc}}=\sum_{j=1}^{N_{\mathrm{dc}}} k_{j, \mathrm{dc}}$

$\omega_{\mathrm{ac}}^{*}=\sum_{k=1}^{N_{\mathrm{ac}}}\left(\omega_{k, \mathrm{ac}}^{*} k_{k, \mathrm{ac}}\right) / \sum_{k=1}^{N_{\mathrm{ac}}} k_{k, \mathrm{ac}}$

$P_{\mathrm{s}, \mathrm{ac}}=\sum_{k=1}^{N_{\mathrm{ac}}} P_{\mathrm{s} k, \mathrm{ac}}$

$k_{\mathrm{ac}}=\sum_{k=1}^{N_{\mathrm{ac}}} k_{k, \mathrm{ac}}$

\subsection{Local control design for DC-AC and DC-DC interlinking MG converters}

In this section, a novel unified control for DC-AC and DC-DC interlinking converters are proposed. In terms of the basic operation modes and control functions presented briefly in Sections 2.2 and 3.1, detailed requirements for the proposed unified control design are as follows.

1) Requirement 1: DC-AC and DC-DC interlinking converters should be able to receive power dispatch settings from upper central control systems to achieve the global optimal operation target.

2) Requirement 2: under unexpected or large power disturbances in the MG sub-systems (e.g., DG output variations, transient load disturbances of loads), with the proposed unified controls of these interlinking converters, automatically coordinated power control and mutual support among the sub-systems should be 
attained with only locally measured signals, which can improve DC voltage (AC frequency) stability.

3) Requirement 3: furthermore, with the proposed control, MG interlinking converters can realize seamless transition among power dispatching mode, common DC bus voltage control mode, and MG support mode, without communication and control system switching.

The main contribution of this work is our proposed unified control for interlinking converters, shown in Fig. 4. The proposed unified control contains both outer loop and inner loop: (1) outer loop, the main function of the outer loop control is to meet requirements 1 and 2 and to generate power references for the inner loop; (2) inner loop, the inner loop is mainly used to track power references from the outer loop and to meet requirement 3 .

For outer loop control, in order to realize power dispatch tracking mentioned in requirement 1 , simple proportionalintegral (PI) controllers are used, as follows.

$\left\{\begin{aligned} \Delta P_{\mathrm{dp}, \mathrm{ac}} & =\left(P_{\mathrm{IC}, \mathrm{ac}}^{*}-P_{\mathrm{IC}, \mathrm{ac}}\right) G_{\mathrm{dp}, \mathrm{ac}}(s) \\ \Delta P_{\mathrm{dp}, \mathrm{dc}} & =\left(P_{\mathrm{IC}, \mathrm{dc}}^{*}-P_{\mathrm{IC}, \mathrm{dc}}\right) G_{\mathrm{dp}, \mathrm{dc}}(s)\end{aligned}\right.$ where $P_{\mathrm{IC}, \mathrm{ac}}^{*}$ and $P_{\mathrm{IC}, \text { ac }}$ are power dispatch setting and actual output of the DC-AC interlinking converter and the AC MG, respectively; $P_{\mathrm{IC}, \mathrm{dc}}^{*}$ and $P_{\mathrm{IC}, \mathrm{dc}}$ are power dispatch setting and actual output of the DC-DC converter and DC MG interlinking converter, respectively; $G_{\mathrm{dp}, \mathrm{ac}}(s)$ and $G_{\mathrm{dp}, \mathrm{dc}}(s)$ are PI controller-based transfer functions.

For automatically coordinated power control and mutual support among sub-systems, a novel power support control law is designed as follows. See Fig. 4.

When there is a power disturbance in any sub-system, it causes varying corresponding DC voltages or AC frequency. Via the control in (13), automatic transient power response can be realized to enhance the anti-disturbance performance and mutual power support capability among sub-regions, and to further improve DC voltage (AC frequency) control stability.

$$
\left\{\begin{array}{l}
\Delta P_{\mathrm{ap}, \mathrm{ac}}=-k_{\mathrm{ac}}\left(\omega_{\mathrm{ac}}^{*}-\omega_{\mathrm{ac}}\right)+\alpha k_{\mathrm{MV}}\left(u_{\mathrm{MV}}^{*}-u_{\mathrm{MV}}\right) \\
\Delta P_{\mathrm{ap}, \mathrm{dc}}=-k_{\mathrm{dc}}\left(u_{\mathrm{dc}}^{*}-u_{\mathrm{dc}}\right)+\beta k_{\mathrm{MV}}\left(u_{\mathrm{MV}}^{*}-u_{\mathrm{MV}}\right)
\end{array}\right.
$$

where $\alpha$ and $\beta$ in (13) are transient power support coefficients, used to measure the strength of the power support

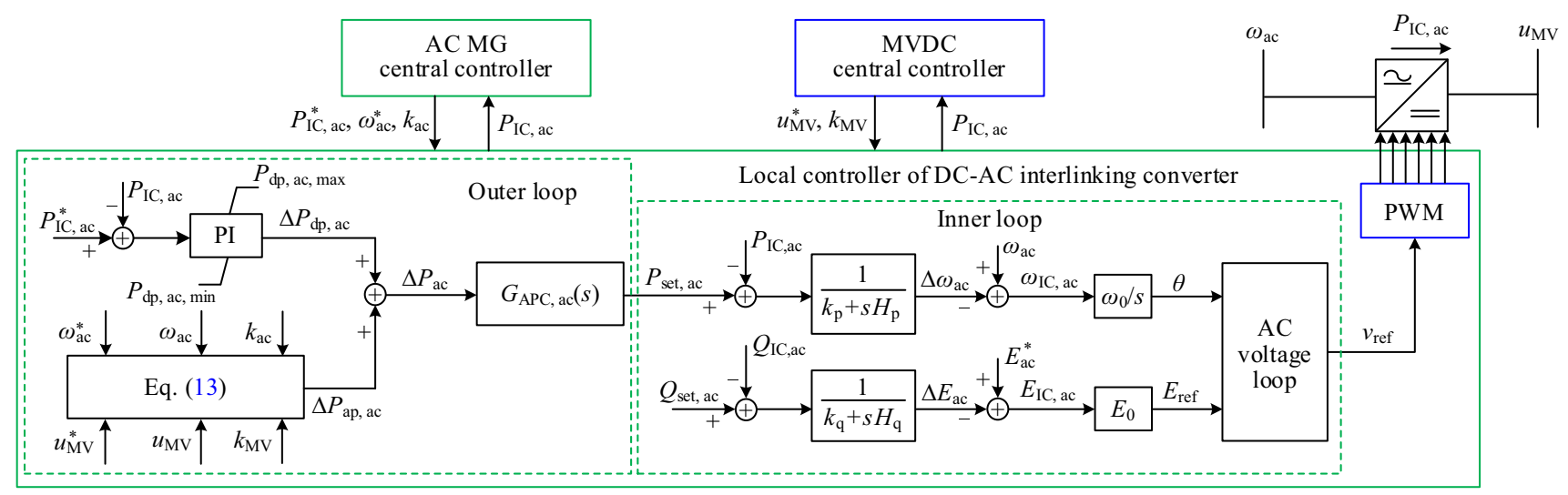

(a)

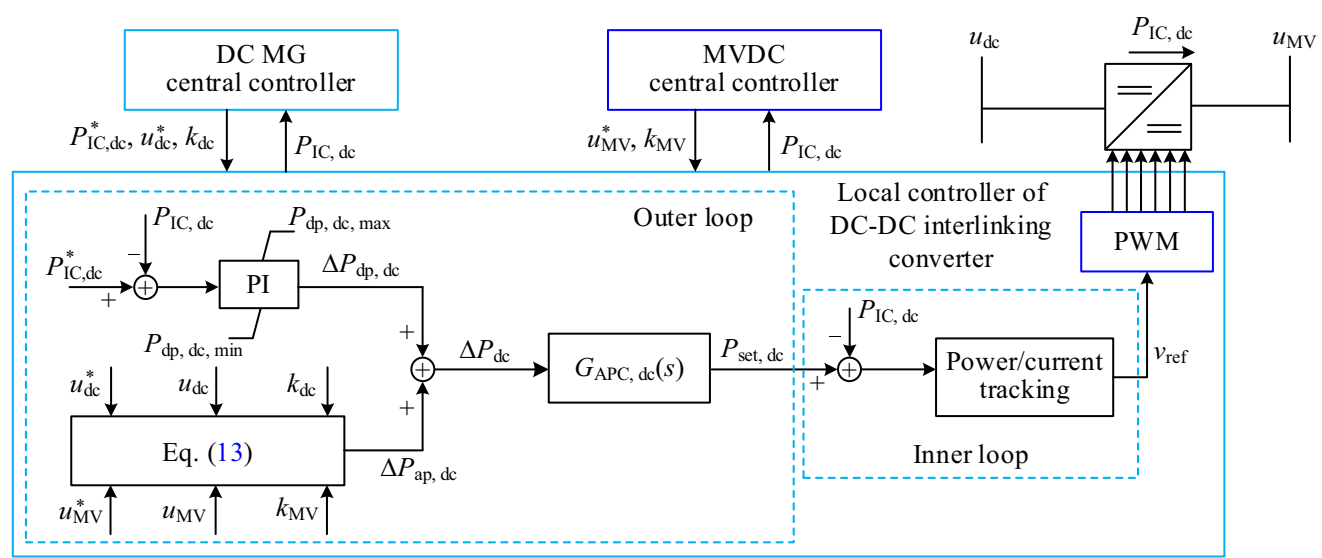

(b)

Fig. 4 Unified control for the interlinking converters 
capability of sub-systems during transient power disturbances.

With (12) and (13), power references for the inner loop ( $P_{\text {set,ac }}$ and $\left.P_{\text {set,dc }}\right)$ can be obtained as:

$\left\{\begin{array}{l}P_{\mathrm{set}, \mathrm{ac}}=\left(\Delta P_{\mathrm{dp}, \mathrm{ac}}+\Delta P_{\mathrm{ap}, \mathrm{ac}}\right) G_{\mathrm{APC}, \mathrm{ac}}(s) \\ P_{\mathrm{set}, \mathrm{dc}}=\left(\Delta P_{\mathrm{dp}, \mathrm{dc}}+\Delta P_{\mathrm{ap}, \mathrm{dc}}\right) G_{\mathrm{APC}, \mathrm{dc}}(s)\end{array}\right.$

where $G_{\mathrm{APC}, \mathrm{ac}}(s)$ and $G_{\mathrm{APC}, \mathrm{dc}}(s)$ are PI controllers.

To achieve seamless transition of different operation modes, DC-AC and DC-DC converters adopt inertia mimicry based droop control [20] and direct power/current tracking control as their inner loops, respectively.

It can be summarized as:

1) When all sub-systems work during normal operation, the DC-AC and DC-DC interlinking converters can track the power dispatch settings using the control in (12). Automatic transient power support among subsystems can be realized by using the control in (13).

2) When the actual operation mode of the MVDC changes, as mentioned in Section 2.2, the transmission power of the related interlinking converter cannot be further dispatched. Thus, the interlinking converter must support the faulty sub-system and stabilize the $\mathrm{DC}$ voltage or AC frequency. In this scenario, the control loop, shown in (12), will reach its limiting threshold automatically and will lose its power dispatch control capability. However, with (13) and the inner loop, the related interlinking converter will smoothly change to MG support mode or medium DC voltage control mode, achieving seamless transition without operation mode detection, communications, and control system switching.

\subsection{Central control functions of each sub-system}

The main functions of each sub-system's central control system (Fig. 2) are summarized as follows.

1) $\mathrm{DC}$ voltage (AC frequency) recovery control

Because slack terminals adopt DC voltage (AC frequency) droop control, as shown in Fig. 3, the DC voltage (AC frequency) will change with operation point variation, affecting power supply quality and stability when the DC voltage (AC frequency) deviates from its rated value. $\mathrm{DC}$ voltage (AC frequency) recovery control $[21,22]$ is always necessary in a central control system with low-bandwidth communication.

2) Optimal interlinking power dispatching

The MVDC may have different optimal operation objectives in actual applications, such as load sharing among multiple feeders of the AC distribution system, loss minimization-based optimal power-flow control, and optimal bidding strategy for MGs in distribution markets [23, 24]. Distributed cooperation-based optimal control [25] among central control systems shown in Fig. 2 can achieve related optimal targets. The key work of optimal interlinking power dispatching is to determine the power settings of DC-AC and DC-DC converters of MGs, shown as $P_{\mathrm{IC}, \mathrm{ac}}^{*}$ and $P_{\mathrm{IC}, \mathrm{dc}}^{*}$. For different operation modes, a brief analysis is presented as follows.

1) Model 1

With a certain optimal interlinking power dispatching target adopted for the MVDC (e.g., each MG central controller is used to maximize its net profit according to the current states and predicted results of renewable DGs and loads, operation condition of ESSs, price information from electricity markets, and maximum interlinking power constraint between the MVDC and AC distribution system, etc.), the power dispatch settings of DC-AC and DC-DC converters $P_{\mathrm{IC}, \mathrm{ac}}^{*}$ and $P_{\mathrm{IC}, \mathrm{dc}}^{*}$ can be determined first.

In terms of power balancing, the total interlinking power between the MVDC and AC distribution system $P_{\mathrm{s}, \mathrm{MV}}$ can be fixed. Then, the MVDC central controller can be used to optimize power sharing of $P_{\mathrm{s}, \mathrm{MV}}$ among multiple DC-AC converters, as shown in Fig. 1, using optimal load-sharing operation targets among multiple AC distribution system feeders or loss minimizationbased optimal power-flow control, etc.

2) Model 2

Compared to Mode 1, the interlinking power between faulty MG and medium-voltage DC bus is determined directly by the output of the faulty MG's power terminal. However, the MG central controller still needs to receive the maximum interlinking power constraint from the MVDC central control system to maintain power balance by load shedding or via renewable DG output curtailments.

3) Model 3

When all MGs work in islanded mode without interlinking power transmission, each MG can operate in autonomous mode with its own optimal operation and stability targets (e.g., transient power balance, DC voltage or AC frequency stability, and energy storage systems charging/discharging management).

When all normal MGs work in interconnected operation mode, the power dispatch settings of DC-AC and DC-DC converters, $P_{\mathrm{IC}, \mathrm{ac}}^{*}$ and $P_{\mathrm{IC}, \mathrm{dc}}^{*}$ can still be determined by coordinated optimal control. However, 
it should be noted that the settings should satisfy the power balance equation: $P_{\mathrm{IC}, \mathrm{ac}}^{*}+P_{\mathrm{IC}, \mathrm{dc}}^{*}=0$.

In the interconnected operation mode, if a faulty MG requires power support, the transmission power through the related interlinked converter will be directly determined by its power terminal output. The faulty $\mathrm{MG}$ can thus be regarded as a power terminal for the normal $\mathrm{MG}$.

Based on the coordinated control strategy shown in Figs. 2-4, distributed optimization control algorithms are compatible with the proposed local controllers. A brief introduction of the framework shown in Fig. 2 is as follows.

1) The central controllers of MCDC, AC MG, and DC MG realize global optimization by using limited data communication and distributed optimization algorithm, and the optimal boundary power are obtained during the given period. The MVDC optimization target optimizes power sharing among multiple DCAC converters, and optimal objectives of AC MG and DC MG can be set to realize minimum daily operating cost. In addition, the alternating direction method of multipliers [26] and generalized Benders decomposition [27] are two popular distributed optimization algorithms that can be applied to solve the global optimization problem in a distributed manner.

2) Based on the optimal boundary power obtained by global optimization, the central controllers of MCDC, AC MG and DC MG can get the optimal operation parameters of local controllers (as shown in Fig. 3) through independent regional optimization.

\section{Verification of proposed coordinated control}

\subsection{Simplified dynamic model}

In order to verify the proposed coordinated control for the MVDC, a simplified dynamic model, including detailed dynamics of DC bus voltages (AC frequency) and local control loops, is built, as shown in Fig. 5. In this simplified dynamic model, dynamic features of medium DC voltage and DC voltage in the DC MG can be expressed as:

$\left\{\begin{array}{l}K_{\mathrm{e}, \mathrm{MV}} \frac{\mathrm{d} u_{\mathrm{MV}}}{\mathrm{d} t}=P_{\mathrm{s}, \mathrm{MV}}+P_{\mathrm{IC}, \mathrm{ac}}+P_{\mathrm{IC}, \mathrm{dc}} \\ K_{\mathrm{e}, \mathrm{dc}} \frac{\mathrm{d} u_{\mathrm{dc}}}{\mathrm{d} t}=P_{\mathrm{s}, \mathrm{dc}}+P_{\mathrm{p}, \mathrm{dc}}-P_{\mathrm{IC}, \mathrm{dc}}\end{array}\right.$

where $K_{\mathrm{e}, \mathrm{MV}}=C_{\mathrm{MV}} U_{\mathrm{B}, \mathrm{MV}}^{2} / P_{\mathrm{B}}$ and $K_{\mathrm{e}, \mathrm{dc}}=C_{\mathrm{dc}} U_{\mathrm{B}, \mathrm{dc}}^{2} / P_{\mathrm{B}}$. In this work, the base value of power is defined as $P_{\mathrm{B}} ; U_{\mathrm{B}, \mathrm{MV}}$ and $U_{\mathrm{B}, \mathrm{dc}}$ are the base values of medium-DC voltage and DC voltage in the DC $\mathrm{MG}$ respectively; $C_{\mathrm{MV}}$ and $C_{\mathrm{dc}}$ represent the equivalent capacitance in the medium voltage DC bus and the DC MG, respectively.

As seen in Fig. 5, the AC MG mathematic model uses different expressions for different operation modes.

1) When the slack terminal in the AC MG is normal, the AC frequency (defined as $\omega_{\mathrm{ac}}$ ) is dominated by the local control of the slack terminal, shown in Fig. 3c, and the output dynamic of the DC-AC interlinking converter $P_{\mathrm{IC}, \text { ac }}$ is determined by its control system, shown in Fig. 4a.

2) If the slack terminal in the $\mathrm{AC}$ MG becomes unavailable, the AC frequency dynamic will be dominated by the unified control of the DC-AC interlinking converter shown in Fig. 4a, and the interlinking power $P_{\text {IC,ac }}$ will be directly determined by the output of the AC MG's power terminal. In Fig. 5, $G_{\mathrm{pll}}(s)$ is a firstorder inertial loop based phase-lock loop.

In Fig. 5, only one aggregated slack terminal is considered for each subsystem. Droop control and DC voltage control loop were considered, and an inner loop was simplified to a first-order inertial loop. Similarly, for the proposed unified control of DC-AC and DC-DC interlinking converters, dynamic feature of parameters used in Fig. 5 are listed in Table 1. For simplified analysis and validation of the proposed primary control of the considered MVDC: (1) power dispatch settings for the interlinking converters $\left(P_{\mathrm{IC}, \mathrm{ac}}^{*}\right.$ and $\left.P_{\mathrm{IC}, \mathrm{dc}}^{*}\right)$ are tuned directly tuned to simulate coordinated optimal controls among all regions; (2) during simulation process, subsystem faults and operation mode changes are simulated by setting the output of the related slack terminal to zero, (assume that $P_{\mathrm{s}, \mathrm{MV}}=0$, or $P_{\mathrm{s}, \mathrm{ac}}=0$, or $P_{\mathrm{s}, \mathrm{dc}}=0$ ), which is used to verify a seamless transition capability of the proposed unified control of interlinking MG converters.

\subsection{Simulation validations}

1) Case 1

With traditional controls, constant power dispatch is always adopted for MG interlinking converters. Thus, control performance of the traditional method and the proposed control are compared under normal power disturbances in AC and DC MGs. Figure 6a, b show comparisons of DC voltage and power in the DC MG. Comparisons of $\mathrm{AC}$ frequency and power in the $\mathrm{AC}$ MG are shown in Fig. 7a, b. Figure 8 shows the results of the medium voltage DC bus.

In simulations, when $t<2 \mathrm{~s}$, the power terminals outputs of AC and DC MGs were $P_{\mathrm{p}, \mathrm{ac}}=1.0$ and $P_{\mathrm{p}, \mathrm{dc}}$ $=-1.0$. Control of DC-AC and DC-DC interlinking converters of MGs was enabled at $t=2 \mathrm{~s}$, with power 


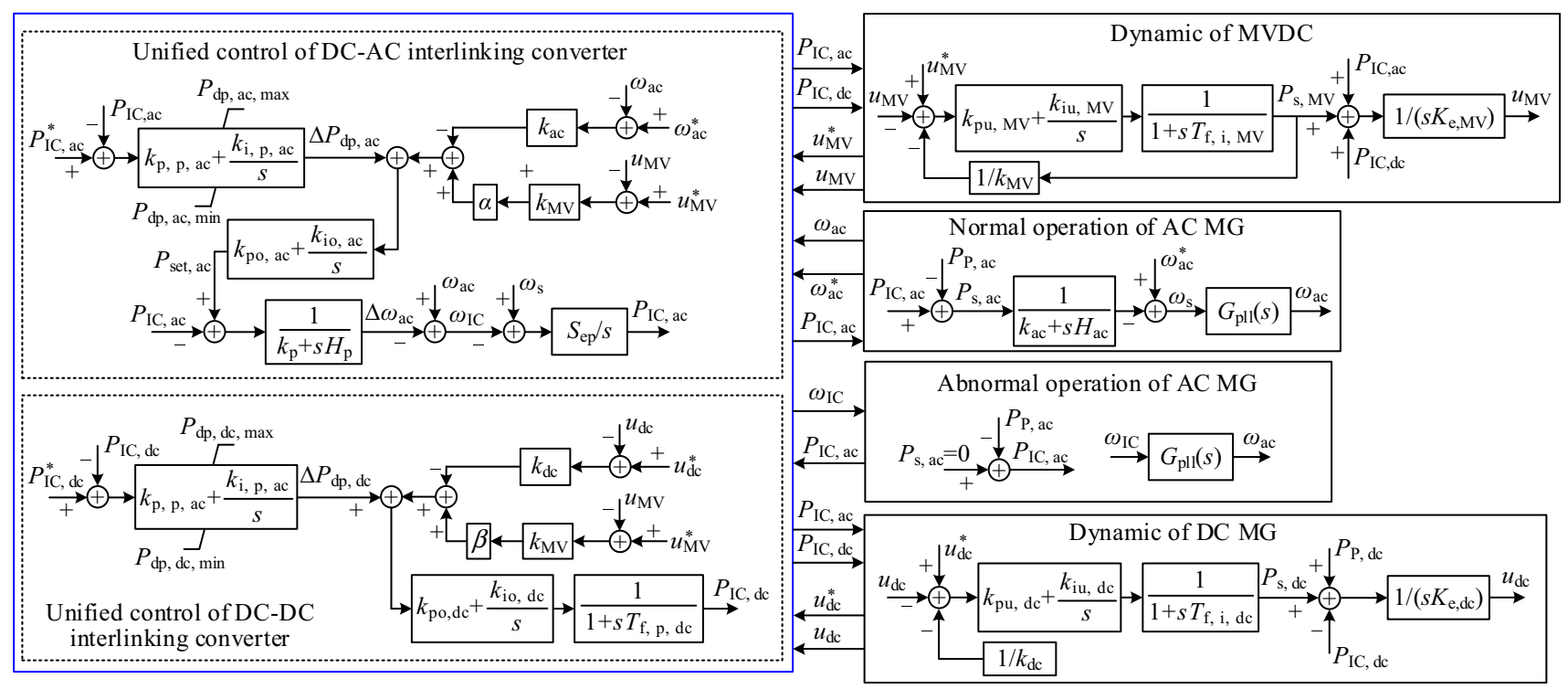

Fig. 5 Simplified dynamic model

Table 1 Parameters for simplified dynamic model

\begin{tabular}{|c|c|c|c|}
\hline Sub region & Dynamic loop & Parameter & Value \\
\hline \multirow[t]{4}{*}{ Medium voltage DC bus region } & DC bus dynamic & $K_{\mathrm{e}, \mathrm{MV}}$ & 4 \\
\hline & Droop control & Voltage setting $u_{\mathrm{MV}}^{*}$, droop gain $k_{\mathrm{MV}}$ & 1,20 \\
\hline & DC voltage loop & PI control $k_{\mathrm{pu}, \mathrm{MV}}, k_{\mathrm{iu}, \mathrm{MV}}$ & 5,200 \\
\hline & Inner loop & $T_{\mathrm{f}, \mathrm{i}, \mathrm{MV}}$ & $1 \mathrm{~ms}$ \\
\hline \multirow[t]{2}{*}{$\mathrm{AC} \mathrm{MG}$} & Droop control & Frequency setting $\omega_{\mathrm{ac}}^{*}$, droop gain $k_{\mathrm{ac}}$, inertia $H_{\mathrm{ac}}$ & $1,20,0.1 \mathrm{~s}$ \\
\hline & PLL $G_{\mathrm{pll}}(\mathrm{s})$ & Time constant $T_{\mathrm{pll}}$ & $1 \mathrm{~ms}$ \\
\hline \multirow[t]{4}{*}{ DC MG } & DC bus dynamic & $K_{\mathrm{e}, \mathrm{dc}}$ & 0.01 \\
\hline & Droop control & Voltage setting $u_{\mathrm{dc}}^{*}$, droop gain $k_{\mathrm{dc}}$ & 1,20 \\
\hline & DC voltage loop & PI control $k_{\mathrm{pu}, \mathrm{dc}}, k_{\mathrm{iu}, \mathrm{dc}}$ & 5,100 \\
\hline & Inner loop & $T_{\mathrm{f}, \mathrm{i}, \mathrm{dc}}$ & $1 \mathrm{~ms}$ \\
\hline \multirow[t]{5}{*}{ DC-AC interlinking converter } & Power dispatch loop & PI control $k_{\mathrm{p}, \mathrm{p}, \mathrm{ac}}, k_{\mathrm{i}, \mathrm{p}, \mathrm{ac}}$ & 2,10 \\
\hline & Power support coefficient & $\alpha$ & 1 \\
\hline & Power tracking & PI control $k_{\mathrm{po}, \mathrm{ac}}, k_{\mathrm{io}, \mathrm{ac}}$ & $0.5,10$ \\
\hline & Droop control & Droop gain $k_{\mathrm{p}}$, inertia $H_{\mathrm{p}}$ & $20,0.1 \mathrm{~s}$ \\
\hline & Inner loop & $S_{\mathrm{ep}}$ & 1000 \\
\hline \multirow[t]{4}{*}{ DC-DC interlinking converter } & Power dispatch loop & PI control $k_{\mathrm{p}, \mathrm{p}, \mathrm{dc}}, k_{\mathrm{i}, \mathrm{p}, \mathrm{dc}}$ & $2.5,10$ \\
\hline & Power support coefficient & $\beta$ & 1 \\
\hline & Power tracking & PI control $k_{\mathrm{po}, \mathrm{dc}}, k_{\mathrm{io}, \mathrm{dc}}$ & $0.5,10$ \\
\hline & Droop control & $T_{\mathrm{f}, \mathrm{p}, \mathrm{dc}}$ & $1 \mathrm{~ms}$ \\
\hline
\end{tabular}

dispatch settings to be $P_{\mathrm{IC}, \mathrm{ac}}^{*}=0.8$ and $P_{\mathrm{IC}, \mathrm{dc}}^{*}=-0.5$. Power terminal output in the DC MG was changed to $P_{\mathrm{p}, \mathrm{dc}}=-0.5$ at $t=3 \mathrm{~s}$, and again reduced to $P_{\mathrm{p}, \mathrm{dc}}=$ -1.0 at $t=4 \mathrm{~s}$. The output of the power terminal in the AC MG was reduced to $P_{\mathrm{p}, \mathrm{ac}}=0.5$ at $t=5 \mathrm{~s}$, and again changed to $P_{\mathrm{p}, \text { ac }}=1.0$ at $t=6 \mathrm{~s}$.

As seen from simulation results shown in Figs. 6 and 7 , when there was a power disturbance in the DC or AC MG, transmissions of interlinking converters of MGs (referred to $P_{\mathrm{IC}, \mathrm{dc}}$ and $P_{\mathrm{IC}, \mathrm{ac}}$ ) remained unchanged with traditional constant power dispatch control. While with the proposed unified control, these DC-DC and DC-AC interlinking converters can provide transient power support for the disturbed MG, which could reduce variation of DC voltage and $\mathrm{AC}$ frequency, and enhance system stability under transients. After 


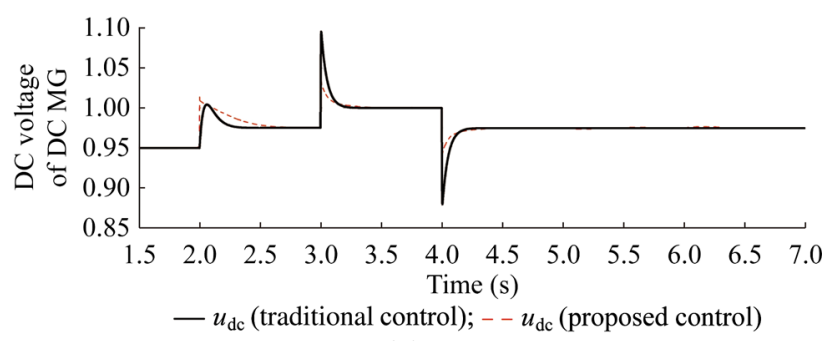

(a) DC voltage

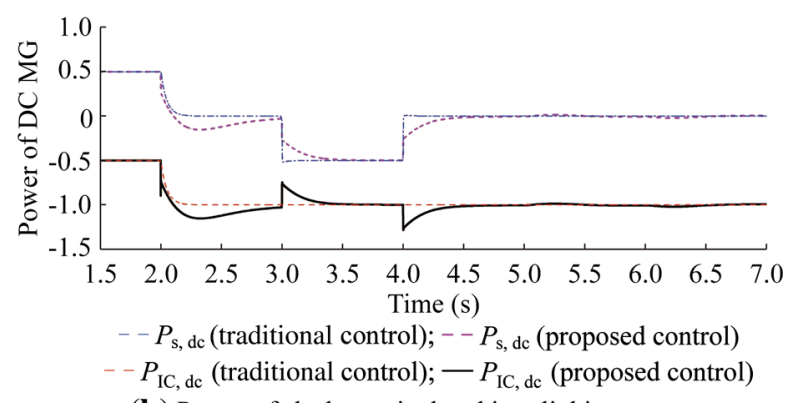

(b) Power of slack terminal and interlinking converter

Fig. 6 Simulation results of DC MG in case 1

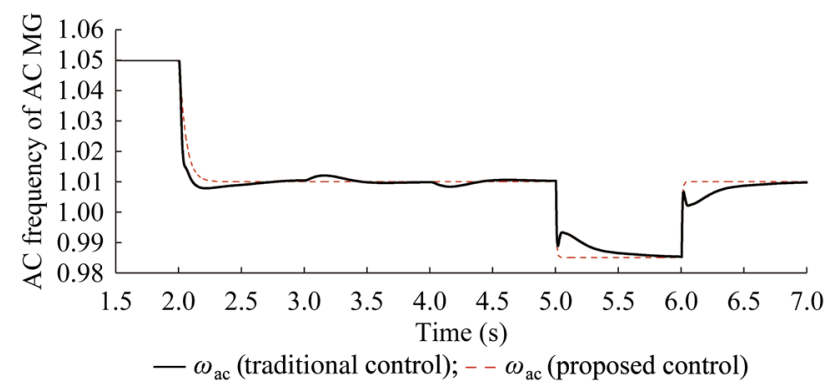

(a) AC frequency

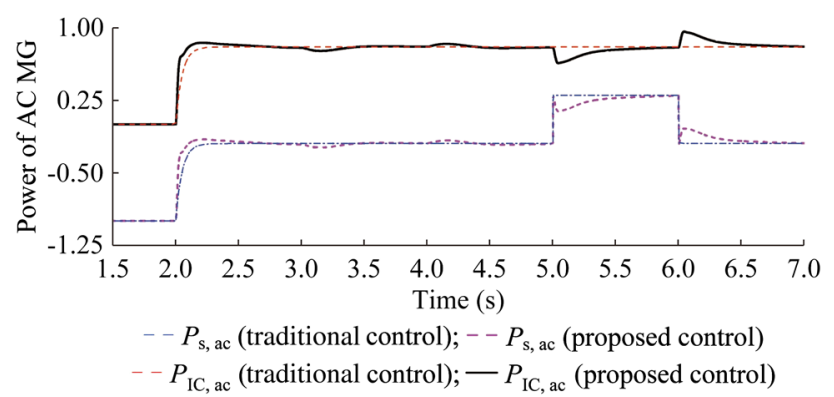

(b) Power of slack terminal and interlinking converter

Fig. 7 Simulation results of AC MG in case 1

transients, these interlinking converters of MGs could still track their power dispatch settings accurately. With the proposed unified control, coordinated power control and mutual support among sub-systems could be realized automatically.

2) Case 2

This case is mainly used to verify the proposed control under following transient scenarios: (1) online

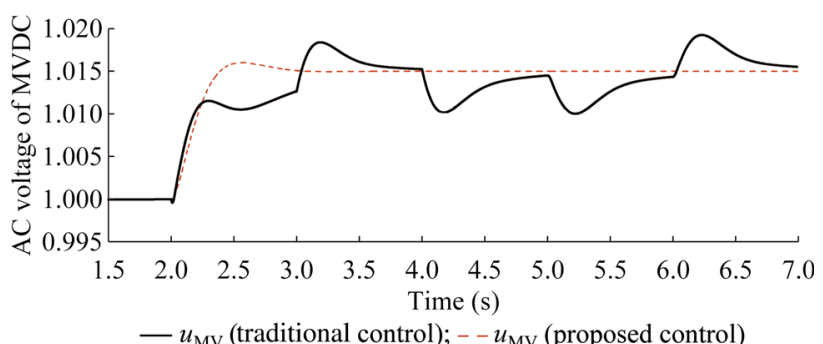

$-u_{\mathrm{MV}}$ (traditional control); $--u_{\mathrm{MV}}$ (proposed control)

(a) DC frequency

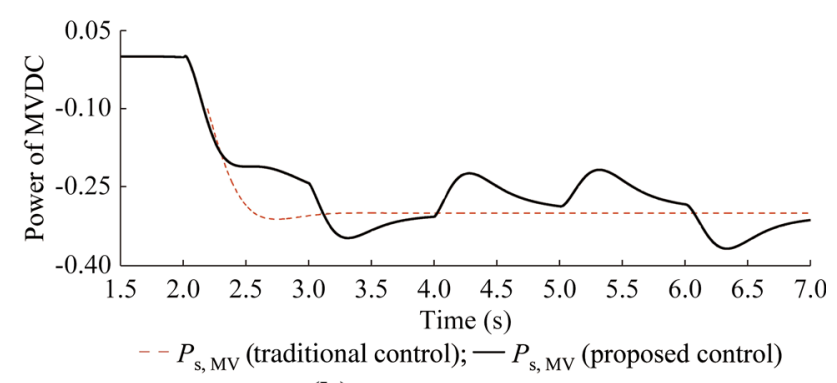

(b) Power of slack terminal

Fig. 8 Simulation results of MVDC in case 1

tuning of power dispatch settings of interlinking converters of MGs in Mode 1; (2) operation of MVDC changing from Mode 1 to Mode 2 by stopping the slack terminals in the MGs. Figure $9 a, b$ shows the power of interlinking converters and outputs of power dispatch loop of the DC-AC and DC-DC interlinking converters respectively. Actual DC voltages (AC frequency) and outputs of slack terminal in each subsystem are shown in Fig. 10a, b, respectively.

The detailed simulation process is as follows. When $t<6.0 \mathrm{~s}$, outputs of power terminals in AC and DC MGs were $P_{\mathrm{p}, \mathrm{ac}}=1.0$ and $P_{\mathrm{p}, \mathrm{dc}}=0.5$. The unified control-based interlinking converters of MGs worked with initial power dispatch settings to $P_{\mathrm{IC}, \mathrm{ac}}^{*}=0.1$ and $P_{\mathrm{IC}, \mathrm{dc}}^{*}=-0.2$. At $t=6 \mathrm{~s}$. Power dispatch settings of DC-AC and DC-DC interlinking converters of MGs were changed to $P_{\mathrm{IC}, \mathrm{ac}}^{*}=-0.1$ and $P_{\mathrm{IC}, \mathrm{dc}}^{*}=0.1$, which was used to simulate coordinated optimal control. Then, AC and DC MG slack terminals became unavailable at $t=7.5 \mathrm{~s}$ and at $t=10 \mathrm{~s}$, respectively. It can be seen from the simulation results shown in Figs. 9 and 10 that, in normal operation mode (referred to $t<7.5 \mathrm{~s}$ ), DC-AC and DC-DC interlinking converters accurately tracked the power dispatch settings. When the slack terminal of a MG became unavailable, the power dispatching loop of the related interlinking converter reached its limited value, shown in Fig. 9b. The maximum and minimum values were set as 2 and -2 respectively in this simulation, which had to be carefully designed for actual applications. As expected, the interlinking converters were seamlessly 


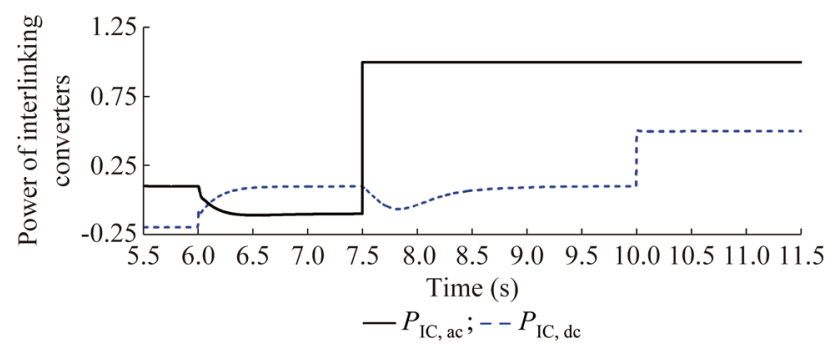

(a) Power of interlinking converters

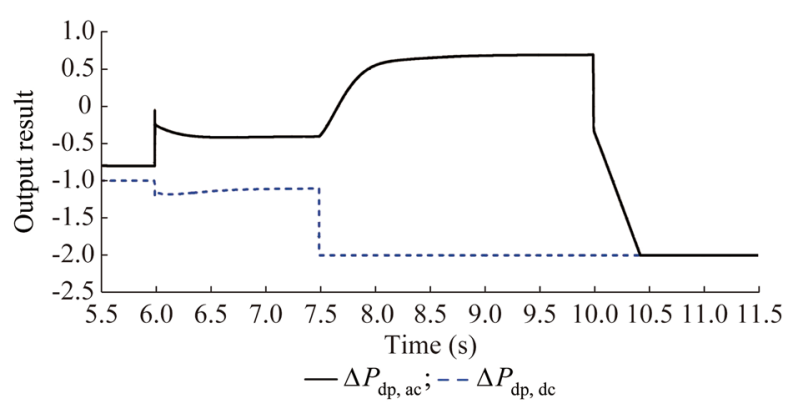

(b) Output results of power dispatch loop

Fig. 9 Simulation results of power of interlinking converters and output results of power dispatch loop in case 2

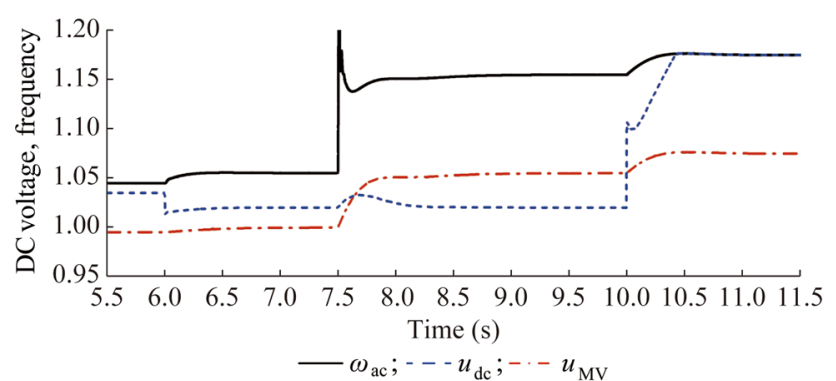

(a) DC voltages and AC frequency

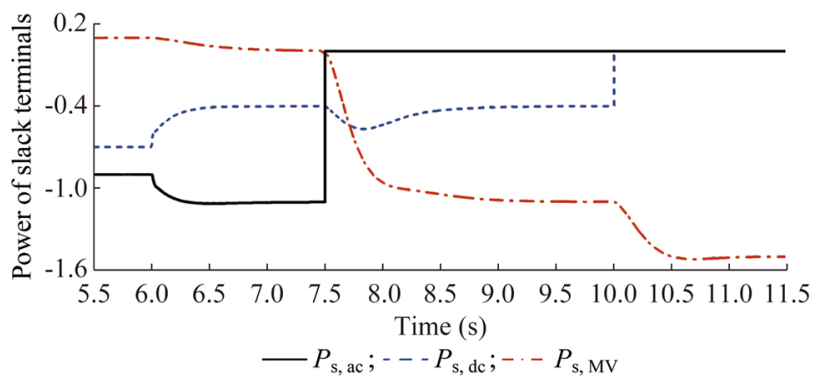

(b) Power of slack terminals

Fig. 10 Simulation results of DC voltages and AC frequency and power of slack terminals in case 2

changed to MG support control with the proposed unified control without operational mode detection, communication, and control system switching.

3) Case 3

This case verifies the performance of the proposed coordinated control when the MVDC's operation

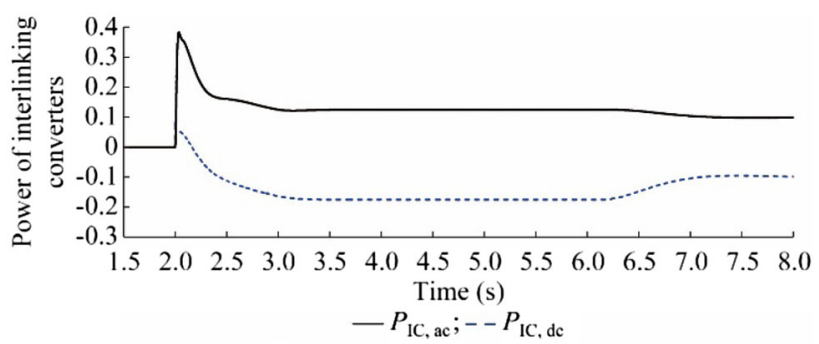

(a) Power of interlinking converters

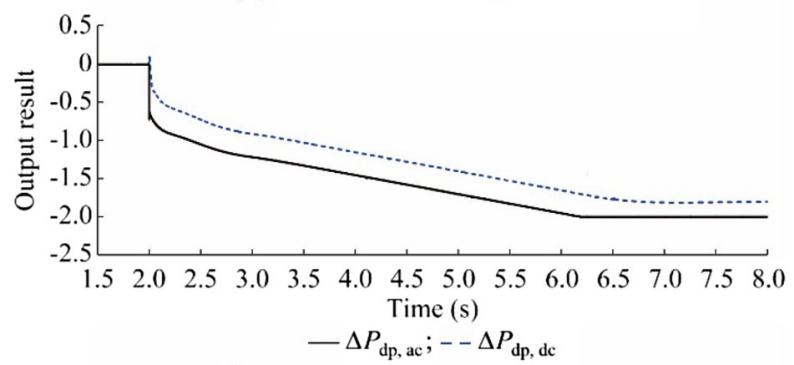

(b) Output results of power dispatch loop

Fig. 11 Simulation results of power of interlinking converters and output results of power dispatch loop in case 3

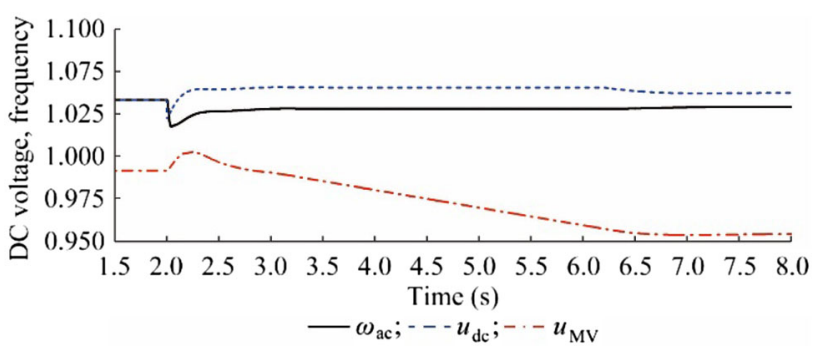

(a) DC voltages and AC frequency

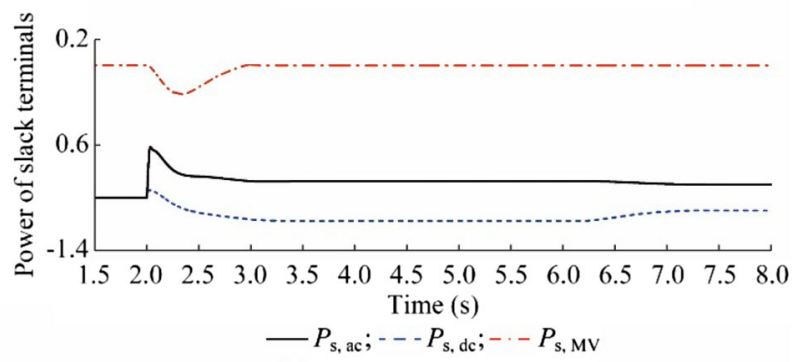

(b) Power of slack terminals

Fig. 12 Simulation results of DC voltages and AC frequency and power of slack terminals in case 3

mode changes from Mode 1 to Mode 3. Figure 11 shows power of interlinking converters and outputs of power dispatch loop of the DC-AC and DC-DC interlinking converters respectively. Actual DC voltages (AC frequency) and outputs of slack terminals in each subsystem are shown in Fig. 12, respectively.

When $t<2.0 \mathrm{~s}$, power terminals outputs in $\mathrm{AC}$ and DC MGs were $P_{\mathrm{p}, \mathrm{ac}}=1.0$ and $P_{\mathrm{p}, \mathrm{dc}}=1.0$, and the DC- 
$\mathrm{AC}$ and DC-DC interlinking converters of MGs were both in standby state. At $t=2.0 \mathrm{~s}$, the unified control of DC-AC and DC-DC was enabled, with initial power dispatch settings to be $P_{\mathrm{IC}, \mathrm{ac}}^{*}=0.1$ and $P_{\mathrm{IC}, \mathrm{dc}}^{*}=-0.2$. The slack terminal in the medium-voltage DC bus (referred to the DC-AC converter between the MVDC and AC distribution system) became unavailable at $t=$ 3 s. From the simulation results, via the proposed control, the MVDC transferred stably to mutual support of AC and DC MGs (Mode 3) from Mode 1, without communication, and without controller switching.

\section{Conclusion}

A novel coordinated control for a typical MVDC is proposed. Theoretical analysis and simulation results verified that the proposed control realized flexible interlinking power control and automatic transient power support among sub-regions, allowing the MVDC with multiple MGs to be treated as a real unified cluster. More important, seamless operation mode transition of the MVDC was also realized without operation mode detection, communication, and control system switching. This work is expected to provide a new solution for flexible interconnection and operation control of large-scale MG clusters.

For future works, the following aspects are recommended: (1) a more detailed dynamic model should be built for stability analysis and optimization of control parameters; (2) a more accurate simulation platform, including a detailed switching model of power electronics devices, should be built to verify the effectiveness of the proposed coordinated control, moreover, we should also develop a simple experimental validation system; (3) in order to study optimal power dispatching problems among multiple MGs and between MGs and AC distribution systems, a complete model including all the central controllers should be built.

Open Access This article is distributed under the terms of the Creative Commons Attribution 4.0 International License (http:// creativecommons.org/licenses/by/4.0/), which permits unrestricted use, distribution, and reproduction in any medium, provided you give appropriate credit to the original author(s) and the source, provide a link to the Creative Commons license, and indicate if changes were made.

Acknowledgement This work was supported by National Nature Science Foundation of China (No. 51507109, No. 51707128), Science and Technology Project of China Southern Power Grid (No. GZKJQQ00000417).

\section{References}

[1] Wang C, Wang D, Zhou Y (2015) Framework analysis and technical challenges to smart distribution system. Autom Electric Power Syst 39(9):2-9

[2] He X, Zong S, Wu J et al (2014) Technologies of power electronic equipment interconnecting and networking in distribution grids. Proc CSEE 34(29):5162-5170

[3] Wang C, Sun C, Li P et al (2015) SNOP-based operation optimization and analysis of distribtuion networks. Autom Electric Power Syst 39(9):82-87

[4] Wang C, Song G, Li P et al (2016) Research and prospect for soft open point based flexible interconnection technology for smart distribution network. Autom Electric Power Syst 40(22):168-175

[5] Cao W, Wu J, Jenkins $\mathrm{N}$ et al (2016) Benefits analysis of soft open points for electrical distribution network operation. Appl Energy 165:36-47

[6] Song Q, Zhao B, Liu W et al (2013) An overview of research on smart DC distribution power network. Proc CSEE 33(25):9-19

[7] Zhao B, Song Q, Liu W et al (2014) High- frequency-link DC solid state transformers for flexible DC distribution. Proc CSEE 34(25):4295-4303

[8] Alex H, Mariesa LC, Gerald TH et al (2011) The future renewable electric energy delivery and management system: the energy internet. Proc IEEE 99(1):133-148

[9] Li X, Guo L, Wang C et al (2016) Key technologies of DC microgrids: an overview. Proc CSEE 36(1):2-17

[10] Zhao B, Zhao Y, Wang Y et al (2015) Energy internet based on flexible medium-voltage DC distribution. Proc CSEE 35(19):4843-4851

[11] Liu G, Zhao Y, Yuan Z et al (2016) Study on demonstration project technical scheme of VSC-DC distribution system in Shenzhen. South Power Syst Technol 10(4):1-7

[12] Sheng W, Duan Q, Meng X et al (2017) Research on the AC\&DC seamless-hybrid fluent power distribution system following the power electronics evolution. Proc CSEE 37(7): 1877-1888

[13] Sheng W, Li R, Li Y et al (2016) A preliminary study on voltage level sequence and typical network architecture of direct current distribution network. Proc CSEE 36(13):3391-3404

[14] Wang C, Li X, Guo L et al (2014) A nonlinear disturbance observer based DC bus voltage control for a hybrid AC/DC microgrid. IEEE Trans Power Electron 29(11):6162-6177

[15] Dragičević T, Lu X, Vasquez JC et al (2016) A DC microgrids-Part II: a review of power architectures, applications, and standardization issues. IEEE Trans Power Electron 31(5):3528-3549

[16] Shafiee Q, Dragičević T, Vasquez JC et al (2014) Hierarchical control for multiple DC-microgrids clusters. IEEE Trans Energy Convers 29(4):922-933

[17] Lu X, Guerrero JM, Sun K et al (2014) Hierarchical control of parallel AC-DC converter interfaces for hybrid microgrids. IEEE Trans Smart Grid 5(2):683-692

[18] Lu X, Sun K, Josep G et al (2013) DC hierarchical control system for microgrid applications. Trans China Electrotech Soc 28(4):35-42

[19] Guo L, Zhang S, Li X et al (2017) Stability analysis and damping enhancement based on frequency-dependent virtual impedance for DC microgrids. IEEE J Emerg Select Top Power Electron 5(1):338-350 
[20] Lv Z, Sheng W, Zhong Q et al (2014) Virtual synchronous generator and its applications in micro-grid. Proc CSEE 34(16):2591-2603

[21] Lu X, Sun K, Huang L et al (2013) Dynamic load power sharing method with elimination of bus voltage deviation for energy storage systems in DC micro-grids. Proc CSEE 33(16):37-46

[22] Shafiee Q, Guerrero JM, Vasquez JC (2014) Distributed secondary control for islanded microgrids-a novel approach. IEEE Trans Power Electron 29(2):1018-1031

[23] Wang C, Song G, Li P et al (2016) A hybrid optimization method for distribution network operation with SNOP and tie switch. Proc CSEE 36(9):2315-2321

[24] Liu Y, Guo L, Wang C et al (2017) Optimal bidding strategy for microgrids in electricity distribution market. Power Syst Technol 41(8):2470-2477

[25] Nehrir MH, Wang C, Strunz K et al (2011) A review of hybrid renewable/alternative energy systems for electric power generation: configurations, control, and applications. IEEE Trans Sustain Energy 2(4):392-403

[26] Chai Y, Guo L, Wang C et al (2018) Network partition and voltage coordination control for distribution networks with high penetration of distributed PV units. IEEE Trans Power Syst 33(3):3396-3407

[27] Lin C, Wu W, Zhang B et al (2017) Decentralized reactive power optimization method for transmission and distribution networks accommodating large-scale DG integration. IEEE Trans Sustain Energy 8(1):363-373

Lei QIAO received the B.S. degree from Tianjin University in 2002, received the M.S. degree form South China University of Technology in 2005. She is a lecturer with School of Control and Mechanical Engineering, Tianjin Chengjian University. Her current research interests involve smart grid and flexible DC distribution network control.

Xialin LI received the B.Sc. degree and the Ph.D. degree from Tianjin University, Tianjin, China, in 2009 and 2014, respectively. Since 2014, he has been a lecture with the School of Electrical Engineering and Automation, Tianjin University, China. In 2016, under the State Scholarship Fund, he was invited as a Visiting Professor to the Department of Electrical and Computer Engineering, University of Alberta, Canada. His current research interests include the modeling and control of power converters, distributed generation, hybrid ac/dc microgrid, and multi-terminal dc grids (MTDC).

Di HUANG received the B.S. degree in electric engineering from Tianjin University, Tianjin, China, in 2017, and now he is working as a master in electrical engineering in Tianjin University since 2017. His work has been in the flexible DC transmission and distribution system.

Li GUO received the B.Sc. the Ph.D. degree in Electrical Engineering from South China University of Technology in 2002 and 2007, respectively. Dr. Guo is currently a full Professor at Tianjin University. His research interests include the optimal planning and design of microgrid, the coordinated operating strategy of microgrid, and the advanced energy management system.

Yutao XU received the B.S. degree from Northwestern Polytechnical University in 2006, received the M.S. degree from Chongqing University in 2012. He is a Senior Engineer with Electric Power Research Institute of Guizhou Power Grid Co. Ltd. His current research interests are in the areas of flexible DC distribution network control and energy internet.

Zhukui TAN received the M.S. degree from East China Jiaotong University in 1999. He is a Senior Engineer with Electric Power Research Institute of Guizhou Power Grid Co. Ltd. His current research interests are in the areas of flexible DC distribution network control and energy internet.

Chengshan WANG received the B.Sc. degree, the M.Sc. degree and the Ph.D. degree from Tianjin University, Tianjin, China, in 1983, 1985 and 1991, respectively. He became a full professor of Tianjin University in 1996. He has been to Cornell University as a visiting scientist from 1994 to 1996 and to Carneigie Mellon University as a visiting professor from 2001 to 2002. Prof. Wang is the gainer of Fok Ying Tung Fund, Excellent Young Teacher Fund of Education Ministry and a winner of National Science Fund for Distinguished Young Scholars. He is the Chief Scientist of 973 project, "Research on the Key Issues of Distributed Generation Systems" from 2009 to 2013 that was participated by Chinese power engineering scientists from 8 leading institutions. His research is in the area of distribution system analysis and planning, distributed generation system and microgrid, and power system security analysis. 